\title{
Présentation du texte de P. Doyé en français
}

\section{Gilbert Dalgalian}

\section{OpenEdition}

\section{Journals}

Édition électronique

URL : http://journals.openedition.org/esp/427

ISSN : 2532-0319

Éditeur

Centre d'Information sur l'Éducation Bilingue et Plurilingue

Édition imprimée

Date de publication : 1 décembre 2014

Pagination : 25

ISSN : 1127-266X

\section{Référence électronique}

Gilbert Dalgalian, «Présentation du texte de P. Doyé en français », Éducation et sociétés plurilingues [En ligne], 37 | 2014, mis en ligne le 01 octobre 2015, consulté le 23 septembre 2020. URL : http:// journals.openedition.org/esp/427 
Présentation en français

G. DALGaLian

\section{PRÉSENTATION DU TEXTE DE P. DOYÉ EN FRANÇAIS}

\section{Gilbert Dalgalian}

$\mathrm{P}$ eter Doyé (des Universités de Berlin et de Braunschweig) replace le partage du savoir et l'enseignement mutuel au centre de la pédagogie des langues :.

«Le dénominateur commun des pédagogies nouvelles (Decroly, Blonsky, Montessori, Gaudig et Kerschensteiner, Freinet, Malaguzzi et Petersen), c'est le rôle central de l'apprenant et son activité autonome, dont une variante essentielle est l'enseignement mutuel ou «en tandem».

Ce principe, valable dans toutes les disciplines, a une pertinence particulière dans l'apprentissage d'une langue vivante. C'est là que les différences entre élèves plus compétents et élèves moins avancés deviennent un atout pour les deux parties. Si en outre les enfants sont de langue maternelle différente, chacun d'eux bénéficie de la compétence de son vis-à-vis dans sa langue maternelle. Double apprentissage donc.

L'apprentissage en tandem privilégie la meilleure des relations interpersonnelles : la relation à deux, avec ses composantes d'admiration, d'intimité et de solidarité.

Les exemples de tandems en milieu institutionnel sont de plus en plus nombreux. Ils bénéficient des échanges pédagogiques en face à face ou à distance grâce aux nouvelles technologies. Certaines universités (Sheffield, Bochum, Oviedo, Coimbra, Gaudalajara) l'ont inscrit dans leurs curriculae. L'Europaschule de Berlin le pratique systématiquement dans neuf filières bilingues et bi-culturelles. Des jardins d'enfants bilingues l'ont également adopté à Berlin, Kiel, Cologne, Munich et Wolfsburg. C'est la voie optimale d'intégration pour les enfants de migrants, mais aussi la meilleure initiation des petits Allemands à la diversité et aux richesses culturelles. Le tandem, c'est la responsabilité pour l'Autre et l'ouverture à l'Autre.

Quatre illustrations pratiques en classe sont détaillées. Elles mettent en évidence qu'un enfant peut être une source et un transmetteur de langue pour son camarade; que, ce faisant, il n'en devient que plus conscient de sa propre compétence de locuteur natif; qu'enfin les différences de niveaux peuvent être surmontées ensemble et que cela vaut dans tous les domaines du savoir. 